TRANSACTIONS OF THE

AMERICAN MATHEMATICAL SOCIETY

Volume 349, Number 4, April 1997, Pages 1463-1485

S 0002-9947(97)01507-9

\title{
ESSENTIAL LAMINATIONS IN $I$-BUNDLES
}

\author{
MARK BRITTENHAM
}

\begin{abstract}
We show that, with a few familiar exceptions, every essential lamination in an interval-bundle over a closed surface can be isotoped to lie everywhere transverse to the $I$-fibers of the bundle.
\end{abstract}

\section{INTRODUCTION}

In [B1], we showed that an essential lamination in a (closed) Seifert-fibered space always has a 'vertical' or 'horizontal' sublamination, that is, one which can be isotoped either to be transverse to all of the circle fibers of the Seifert-fibering, or to contain every circle fiber that it meets. In addition, if the sublamination is horizontal and contains no compact leaves, then we showed that the entire lamination could be made horizontal.

In this paper we answer the one question left open by these results: what happens if the horizontal sublamination does contain a compact leaf? What we find is that, with some well-known exceptions, we can still make the entire lamination horizontal. This will follow from the following two results, since a closed, connected Seifertfibered space split open along a horizontal surface is a collection of $I$-bundles over closed surfaces.

Theorem 1. Any essential lamination $\mathcal{L}$ in $M$, an $I$-bundle over a closed surface $F$ of genus 1, containing the associated $\partial I$-bundle as leaves, can be extended to a foliation of $M$ (although not necessarily a horizontal, one, i.e., transverse to the I-fibers).

Theorem 2. Any essential lamination in $M$, an I-bundle over a closed surface $F$ of genus $>1$, containing the associated $\partial I$-bundle as leaves, can be isotoped to lie transverse to the I-fibers of $M$. (It therefore can also be extended to a foliation.)

These results are proved using a form of infinite isotopy, as in [B1], [B2], and [B3], which attempts to put the lamination into Haken normal form, using the most standard cell decomposition of an $I$-bundle over a closed surface, namely, the inverse image under the projection of the standard cell decomposition of a closed surface. This is described in Section 2. The isotopy construction is designed to make our essential lamination horizontal, if the process terminates in finite time. In the succeeding sections we give the proofs of the theorems; they amount to analyzing what might occur to keep the isotopy construction from terminating. In the present instance, the cell decomposition is simple enough that we can use a

Received by the editors September 20, 1994 and, in revised form, April 13, 1995.

1991 Mathematics Subject Classification. Primary 55R05, 57R30; Secondary 57M99, 57N10.

Research supported in part by NSF grant \# DMS-9203435 .

(C)1997 American Mathematical Society 
case-by-case analysis to prove that in the first situation we can always extend the lamination to a foliation (in the process, recognizing the lamination as a 'standard' one), and in the second situation we always arrive at a contradiction.

We deal first with the case that $M=F \times I$, where $F$ is a closed orientable surface; the other cases follow quickly, and in a completely analogous way, from the proof of this case. We describe the changes required in Section 6 .

\section{Preliminaries}

We refer the reader to [G-O] and [B1] for basic definitions and constructions concerning essential laminations.

A closed orientable surface $F$ of genus $g$ has a standard cell decomposition, as a single $4 g$-gon with edges labelled (clockwise) in the familiar pattern

$$
a_{1}, b_{1}, a_{1}^{-1}, b_{1}^{-1}, \ldots, a_{g}, b_{g}, a_{g}^{-1}, b_{g}^{-1} .
$$

By taking the Cartesian product of these cells with $I$, we then get a cell decomposition $K$ of $M=F \times I$ with one 3 -cell, $2 g+2$ 2-cells (including one each from top and bottom), $4 g+1$ 1-cells, and two 0-cells; see Figure 1. One of the 1-cells, the 'vertical' one (which projects to the unique 0-cell in the decomposition of the base surface), will play a key role; we denote it by $I_{0}$.

Given an essential lamination $\mathcal{L}$ in $M$, we can make it transverse to this cell decomposition (after possibly pushing the leaves of $\mathcal{L}$ in $\partial M$ into its interior), by choosing a branched surface $B$ carrying $\mathcal{L}$ and making $B$ transverse to the cell decomposition. We can also use this branched surface to determine a 'monogon number' $\epsilon$ for $\mathcal{L}$ w.r.t. the 1-skeleton of $M$; any two points of $\mathcal{L} \cap K^{(1)}$ which are within $\epsilon$ of one another are contained in the same $I$-fiber of $N(B)$.

Non-orientable surfaces have a similar standard cell decomposition; represented as a connected sum of $g$ projective planes, we obtain a decomposition as a single $2 g$-gon with edges labelled clockwise $a_{1}, a_{1}, \ldots, a_{g}, a_{g}$. Crossing this with the interval $I$ gives us a cell decomposition of $F \times I$. $I$-bundles $M$ which are not products have a similar cell decomposition (since every $I$-bundle over a disk is a product), except that when pairs of vertical 2-cells are glued together, some of them are glued with a flip of the $I$-direction.

We assume that our essential lamination is carried by a branched surface. For some laminations $\mathcal{L}$, such as codimension-one foliations, this requires a preliminary splitting of $\mathcal{L}$ along a finite number of leaves. However, if we can show that this split lamination satisfies the conclusions of our two theorems, then the original one does, as well. For, in the first case if the split lamination can be extended to a foliation, this just means that its complementary components can be foliated, and these pieces include all of the complementary components of the original lamination, so the original lamination can be extended to a foliation. In the second case, if the split open lamination can be isotoped to be transverse to the $I$-fibering, then by collapsing the new lamination back to the old one, as in [B1], we see that the original lamination can also be made horizontal.

Any essential lamination $\mathcal{L}$ in an $I$-bundle $M$ which is horizontal w.r.t the $I$ fibering of $M$ must contain a compact leaf; the first leaf that we encounter along an $I$-fiber as we fall in from one of its ends must be compact (see Figure 1). This is because the component of $\mathcal{L} \cap A$ containing this point, where $A$ is the annulus or Möbius band lying over one of our 1-cells in $F$, is a closed loop; anything else would, because $\mathcal{L}$ is horizontal, violate the fact that our point was the first one 


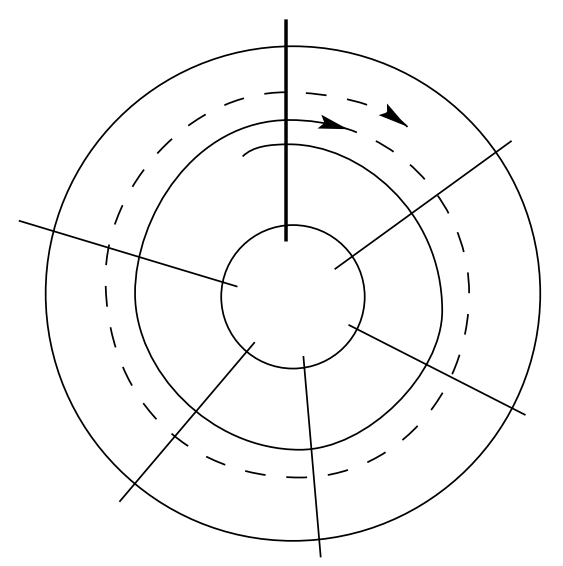

Figure 1

encountered. So the leaf has a cell decomposition identical to our $\partial I$-bundle, hence is compact.

Therefore, the first thing we will establish is that the same is true for every essential lamination in $F \times I$. For any lamination the set of compact leaves is closed; the usual proof of this fact for foliations works without any change. In our case, the proof of [B1] insures us that these compact leaves, all of which can be isotoped to be horizontal in $M$, can be made horizontal simultaneously. All such compact leaves also separate $M$, into two copies of itself. By passing to a sublamination of $\mathcal{L}$, we can therefore assume, without loss of generality, that our essential lamination contains exactly two compact leaves, and that these leaves are the boundary components of $M$. With this, we can then also assume that the branched surface $B$ has the property that $\partial_{h} N(B) \subseteq \mathcal{L}$, and no component of $N(B) \mid \mathcal{L}$ is an $I$-bundle with compact base, by erasing any such components that we happen to have; this amounts to a splitting of $B$. Since each one uses up some of the components of $\partial_{v} N(B)$, there are only finitely many such $I$-bundle components.

\section{THE ISOTOPIES}

Starting with an essential lamination $\mathcal{L}$ in $F \times I$, we now describe a sequence of isotopies of $\mathcal{L}$, which will attempt to make it horizontal w.r.t. the $I$-fibering of $M$. In the present circumstance, making $\mathcal{L}$ horizontal is the same as putting it into Haken normal form $([\mathrm{H}])$ w.r.t the cell decomposition of $M$ that we have built; our 3-cell contains only one type of normal disk, namely the horizontal one. The structure of the isotopy closely follows that of [B3].

Given a essential lamination $\mathcal{L}$ transverse to our cell decomposition of $M=F \times I$, $\mathcal{L}$ meets the boundary of the single 3 -cell $B^{3}$, whose own cell structure looks like a $4 g$-gon crossed with $I$, in a collection of circles, which fall into a finite number of parallel (w.r.t. the cell structure) families. The proof of this is standard. By choosing 2-disks lying parallel to the boundary of the 3 -cell which we then surger $\mathcal{L}$ along, we may assume that all of these loops are bounded by disks of $\mathcal{L} \cap B^{3}$. By throwing away any 2 -spheres that may have been created by these surgeries, we obtain a lamination isotopic to (and which we will still call) $\mathcal{L}$. These disks in $B^{3}$ are unlikely to be in normal form, at this point; if they were, we would be done, 
since they could then be easily isotoped to be transverse to the natural $I$-fibering of the 3 -cell, and so $\mathcal{L}$ could be made horizontal in $M$. The procedure in [B2], for a triangulated 3-manifold (which we are going to adapt), was to go through the 3-simplices one at a time, and surger these 2-disks to make them normal, by a process of ' $\partial$-compression'. Here we have only one 3-cell, so this will not work; anything we push out of the 3-cell is immediately pushed back in (Figure 2a).

We will not worry about this, however. Because we are dealing with a single 3-cell with the structure of a product, and the 2-disks of $\mathcal{L} \cap B^{3}$ do not meet the 'top' and 'bottom' of this 3-cell, these 2-disks can fail to be in normal form in only a very simple way -if a disk crosses the same 1-cell twice, then there is a disk in the boundary of the 3 -cell for us to use as a $\partial$-compressing disk (see Figure $2 b)$. This disk crosses the vertical 1-cells of $\partial B^{3}$ many times, but each such disk contains an 'innermost' disk, which meets the 1-cells only in its boundary. If we focus on pushing these 'obvious' $\partial$-compressing disks instead, then the idea is that we 'should' eventually reach a point where we will be killing the initial two points that we identified.

What we shall do, then, is to order the vertical 1-cells in the boundary of the 3cell (perhaps cyclically), and then do only the $\partial$-compressions that are 'innermost' for that 1-cell; namely those whose compressing disk intersects only our chosen 1-cell. These $\partial$-compressions of course push things out of the 3-cell and then right back in along all of the other 1-cells (possibly immediately creating innermost $\partial$ compressions for the edge we just fixed!), but the point is that we have forced any problems that we once had at our 1-cell to move.

This is how we build our isotopies. We at each stage surger $\mathcal{L}$ and throw away any 2 -spheres, to make $\mathcal{L}$ meet the 3 -cell in disks. Then we push any obvious $\partial$ compressing disks w.r.t one of the vertical 1-cells in the boundary of the 3-cell. By continually working cyclically through the 1-cells, this will create an infinite string of isotopies of $\mathcal{L}$, attempting to put $\mathcal{L}$ into Haken normal form w.r.t. our chosen cell decomposition (and hence make it horizontal). These isotopies $I_{n}$ have the property that the resulting laminations intersect the vertical 1-cell in nested sets, and do not move any point of this intersection that they do not erase.

Now the standard arguments of [B2] allow us to conclude that, first, the intersection of these closed, nested sets is non-empty. For otherwise it is empty at some finite stage (by compactness of the 1-skeleton of $F \times I$ ), implying that our lamination is contained in the complement of the vertical 1-cell. But this is a handlebody,

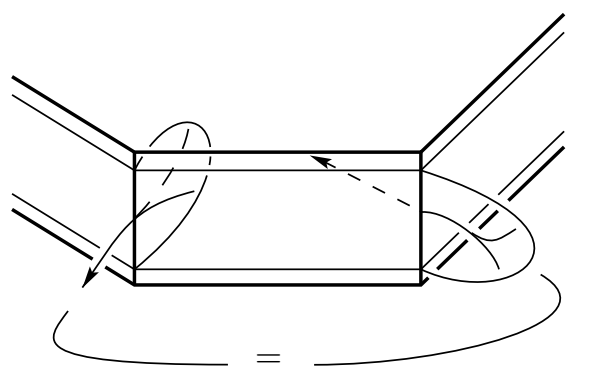

(a)

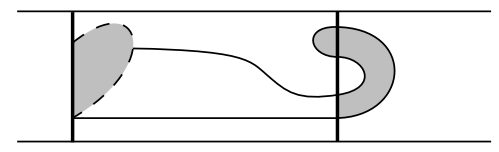

(b)

Figure 2 


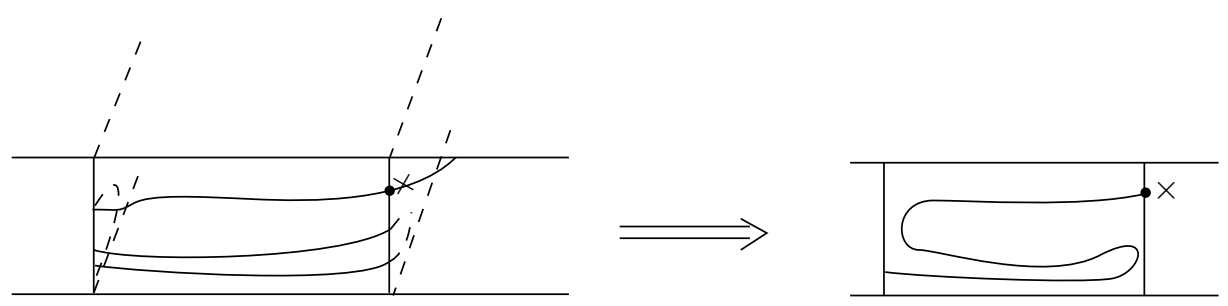

(a)

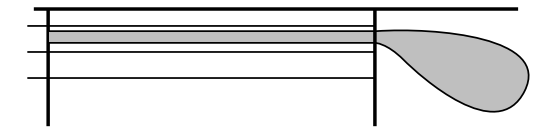

(b)

FiguRE 3

and essential laminations do not live in handlebodies [B1]. We can also conclude that out of this set of stable points for our isotopies, stable normal disks begin to grow. This is because an arc of $\mathcal{L} \cap K^{(2)}$ in one of the vertical 2-cells, which contains a stable point in its boundary, can then only change by 'splicing' (Figure 3a), and therefore must eventually have a stable point as its other endpoint. For otherwise the argument from [B1], [B2] will eventually find an arc in a leaf which together with too short an arc (i.e., length less than the monogon number $\epsilon$ ) in the vertical 1-cell, bounds a disk in $\partial B^{3}$; so the two arcs are homotopic rel endpoints. But this vertical arc must then be in the $I$-fiber of a branched surface carrying $\mathcal{L}$, contradicting [G-O, Theorem 1(d)].

This stable arc must be isotopic to a horizontal one, since otherwise it bounds a $\partial$-compressing disk for the 1-cell that contains its endpoints. But we would then eventually be required to push along this disk, since no previous $\partial$-compression can move the arc in its boundary - its endpoints would have to move - so it will be available for pushing when we cycle around to the 1-cell again, a contradiction. By continuing around the boundary of the 3-cell, we can string together these horizontal arcs until we travel all of the way around the 3-cell. The string must then close up; otherwise, we would instead be forced to continue to string stable (horizontal) arcs together, winding around the 3-cell, until two of them pass within $\epsilon$ of one another. Then, because the string is contained in the boundary of a disk of $\mathcal{L} \cap B^{3}$, there must be a (not necessarily innermost) $\partial$-compressing disk passing $\underline{\text { between }}$ these two stable arcs (see Figure 3b), again contradicting [G-O, Theorem $1(\mathrm{~d})]$.

Once the string closes up, we get a stable horizontal loop in the boundary of our 3-cell; after the next isotopy, it will then bound a stable horizontal disk. So every stable point of the isotopies is eventually contained in a stable normal disk. The union of these stable disks is a horizontal lamination; as in [B2], the only possible problem is that the union is not a closed set in $F \times I$. But, as in [B2], the union can fail to be closed only where two different normal disk types come together in a 3-cell, and since in the present context there is only one normal disk type, this cannot occur. 


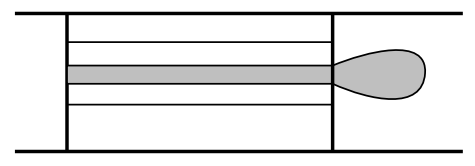

(a)

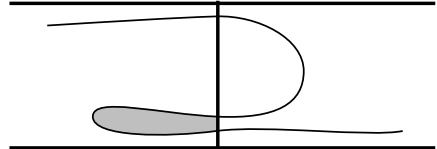

(b)

Figure 4

But because our 3-cell is actually a product, we can conclude that all of these normal disks stabilize at some finite stage of our isotopies. For if not, then at every stage of the isotopies there is some stable point which is contained in an arc of $\mathcal{L} \cap K^{(2)}$ which must later have its other endpoint move. We can, however, wait long enough so that every stable point which is not already in a stable disk lies between two stable disks which are everywhere within $\epsilon$ of one another. This can be arranged by choosing finitely-many stable points in each vertical 1-cell of $B^{3}$, each within $\epsilon$ of its neighbors, and then waiting until they are all contained in stable disks. After that stage of the isotopies, if an arc containing a stable point were to move, we would create a monogon whose vertical boundary had length less than $\epsilon$ (Figure 4a), a contradiction.

Therefore our essential lamination contains a horizontal sublamination, which includes all of the compact leaves of $\mathcal{L}$; ordinary induction on the number of points of intersection of a compact leaf with the vertical 1-cell insures that the entire leaf would eventually be stable. As discussed above in section 1, this sublamination must contain compact leaves, whose union is a closed set, and by cutting down our original lamination to the sublamination which lives between (and includes) two adjacent compact leaves, we may assume that $\mathcal{L}$ contains exactly two compact leaves, which form the boundary of $F \times I$.

If in addition our lamination contains a non-compact horizontal leaf (which must also have stabilized in finite time), then by the argument of [B1, Section 1] we can make the entire lamination horizontal; all other leaves live in an $I$-bundle over a non-compact base, and, since they are carried by a branched surface, are already horizontal near infinity, so can be pulled horizontal. Therefore, we may assume, from now on, that the only leaves of $\mathcal{L}$ which stabilize in finite time are the boundary leaves of $F \times I$; all other leaves, and therefore all other points of intersection of $\mathcal{L}$ with the vertical 1-cell, are unstable and will disappear in the limit.

In particular, eventually all of the points of $\mathcal{L} \cap\left(\right.$ the vertical 1-cell $\left.I_{0}\right)$ which have survived the isotopies up to that point are all within $\epsilon$ of the ends of $I_{0}$, where $\epsilon$ is our monogon number for $\mathcal{L}$. This is because, setting $C_{0}=I_{0} \backslash\left(\epsilon\right.$-nbhd of $\left.\partial I_{0}\right)$, the sets $I_{n}(\mathcal{L}) \cap C_{0}$ are nested, closed subsets of $C_{0}$, with empty intersection (since $\left.\bigcap\left(I_{n}(\mathcal{L}) \cap I_{0}\right)=\partial I_{0}\right)$. So, since $C_{0}$ is compact, $I_{n}(\mathcal{L}) \cap C_{0}=\emptyset$, for some $n$. It then follows that at no time in the remainder of the isotopies does a loop of $\mathcal{L} \cap B^{3}$ cross a vertical 1-cell of $\partial B^{3}$ more than twice; for if it did, two of the points would be within $\epsilon$ of one another (Figure 4b), giving rise to a disk whose boundary consists of an arc in a leaf and a vertical segment in $I_{0}$ of length less than $\epsilon$, contradicting [G-O, Theorem 1(d)]. Therefore, since for each 1-cell in $\partial B^{3}$, the parity of the number of times a disk crosses the 1-cell is the same, there are, for each disk, exactly two possibilities; either it crosses each 1-cell exactly once, and is horizontal, or it crosses 


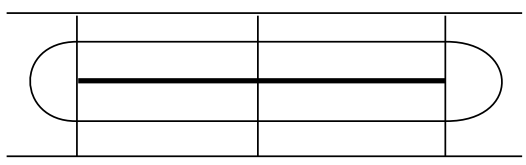

(a)

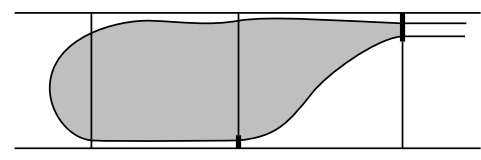

(b)

FiguRE 5

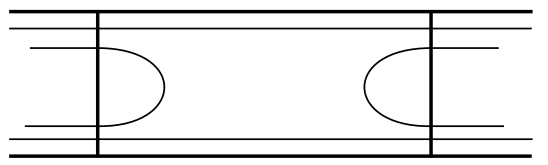

Figure 6

some collection of 1-cells twice and the others not at all, and basically consists of the boundary of a neighborhood of a horizontal arc joining the first edge it crossed to the last (see Figure 5a). Furthermore, if there are any disks of the second type, then every horizontal arc in every vertical 2-cell stays within $\epsilon$ of the top or bottom of $B^{3}$; otherwise, we can again find an arc in $\partial B^{3}$ which, together with a too short arc in $I$, bounds a disk in $\partial B^{3}$ (Figure $5 \mathrm{~b}$ ).

Lemma. There is a stage $I_{k}$ of the isotopies so that, for all successive isotopies $I_{n}$, $n \geq k$, and every vertical 2-cell $R, I_{n}(\mathcal{L}) \cap R$ does not contain $\partial$-compressing arcs on both ends of $R$.

Proof. We can assume, by performing a finite number of the isotopies, that all of the unstable points in $\mathcal{L} \cap I_{0}$ that were at a distance greater than $\epsilon$ from $\partial I_{0}$ have been erased. If we pass through a further full cycle of isotopies, so that we have pushed the obvious $\partial$-compressing disks across each of the 1-cells in $\partial B^{3}$ at least once, we will then find that no vertical 2-cell $R$ in $\partial B^{3}$ has $\partial$-compressing disks on both ends (Figure 6). This is because the arcs of $\mathcal{L}$ in a vertical 2-cell change only by erasure (during surgeries) and by being spliced together along the vertical 1cells of its boundary (during $\partial$-compressions). But $\partial$-compressions must now splice together arcs whose endpoints lie on opposite ends of the vertical 1-cell; otherwise, the ends of the $\partial$-compressing arc lie within $\epsilon$ of one another, a contradiction. But if we imagine gluing together the two sets of $\partial$-compressing arcs from both sides of the same 2-cell-the two vertical 1-cells are, after all, both copies of $I_{0}$-we must get closed loops, because otherwise our lamination has non-trivial holonomy around a null-homotopic loop, a contradiction. It follows that one set of arcs will be 'larger' than the other; when either collection of arcs is pushed (Figure 7), one set vanishes. But once one set is gone, no $\partial$-compression will give both back simultaneously. To get both back would require a $\partial$-compression whose endpoints are either all top or all bottom (Figure 8), because going head-to-head one collection of arcs always swallows the other, leaving only one type (Figure 8).

What we have therefore is very simple behavior in the 3-cell, with an easily catalogued collection of possible patterns for $\mathcal{L} \cap B^{3}$ in $\partial B^{3}$. We carry out this cataloguing in the next section, for the torus. By studying each case we can then see that a lamination which has any of the patterns can be extended to a foliation; 


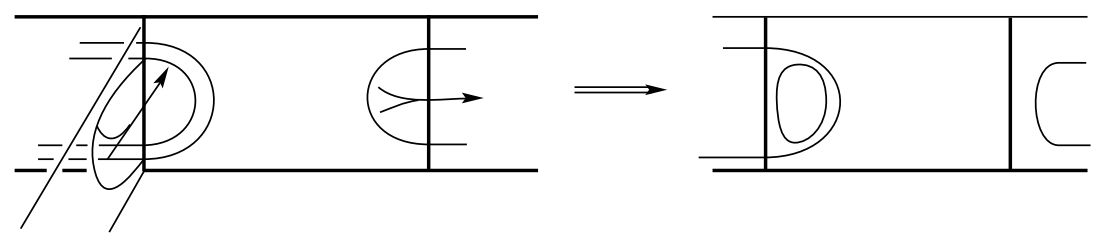

FiguRE 7
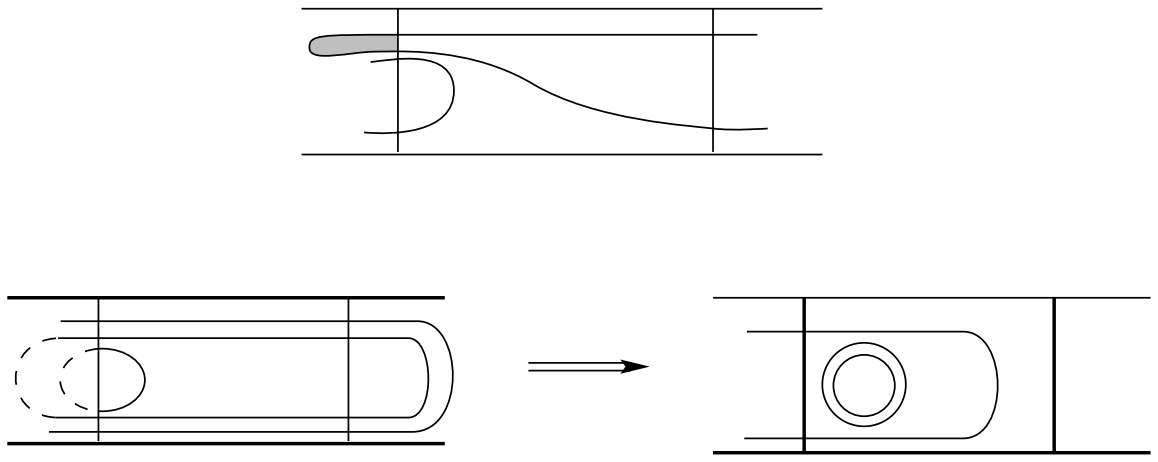

Figure 8

we show this by showing that it is carried by a branched surface $B$ such that $M \backslash N(B)$ consists of product $I$-bundles.

This catalogue will also give us our basic building blocks for understanding the higher-genus case. In that case, however, we find that because we must have at least two of the patterns in succession around $\partial B^{3}$, we can always find a 'compressing disk' for $\mathcal{L}$ which, if 'real', gives an immediate contradiction, while, if not 'real', allows us to show that some other condition of essentiality fails.

\section{The CASE OF A TORUS}

We have $M=T^{2} \times I$, which, with the cell decomposition we have described above, is represented as a cube with opposite vertical faces identified. Given the restrictions on how $\mathcal{L}_{0}=\mathcal{L} \cap B^{3}$ can meet the boundary of this cube, which we have described above, and the fact that $\mathcal{L}_{0}$ must meet the opposite faces in the same way (so that the arcs can be glued together to give $\mathcal{L}$ ), there are, as a consequence only nine $(=3 \times 3)$ patterns of $\partial$-compressing arcs with which $\mathcal{L}_{0}$ can meet $\partial B^{3}$ (and, up to symmetry, only three; although, in anticipation of the next section, we catalogue them all); these are shown in Figure 9. These pictures classify the pattern of 'turns' of $\mathcal{L}$ in $B^{3}$; but, for the last four, each pattern represents three patterns of loops (see Figure 10). In each case these distinct laminations can be carried by a single branched surface (shown in Figure 11b).

The proof of the first theorem consists simply of, in each case, taking the 'obvious' branched surface $B$ carrying our lamination, obtained by collapsing each family of (normally) parallel disks to a single disk, and showing that every component of $M \backslash N(B)$ is an $I$-bundle. Since $N(B) \mid \mathcal{L}$ is also a collection of $I$-bundles, and the $I$-bundle structures can be assumed to agree along their intersection, which is 


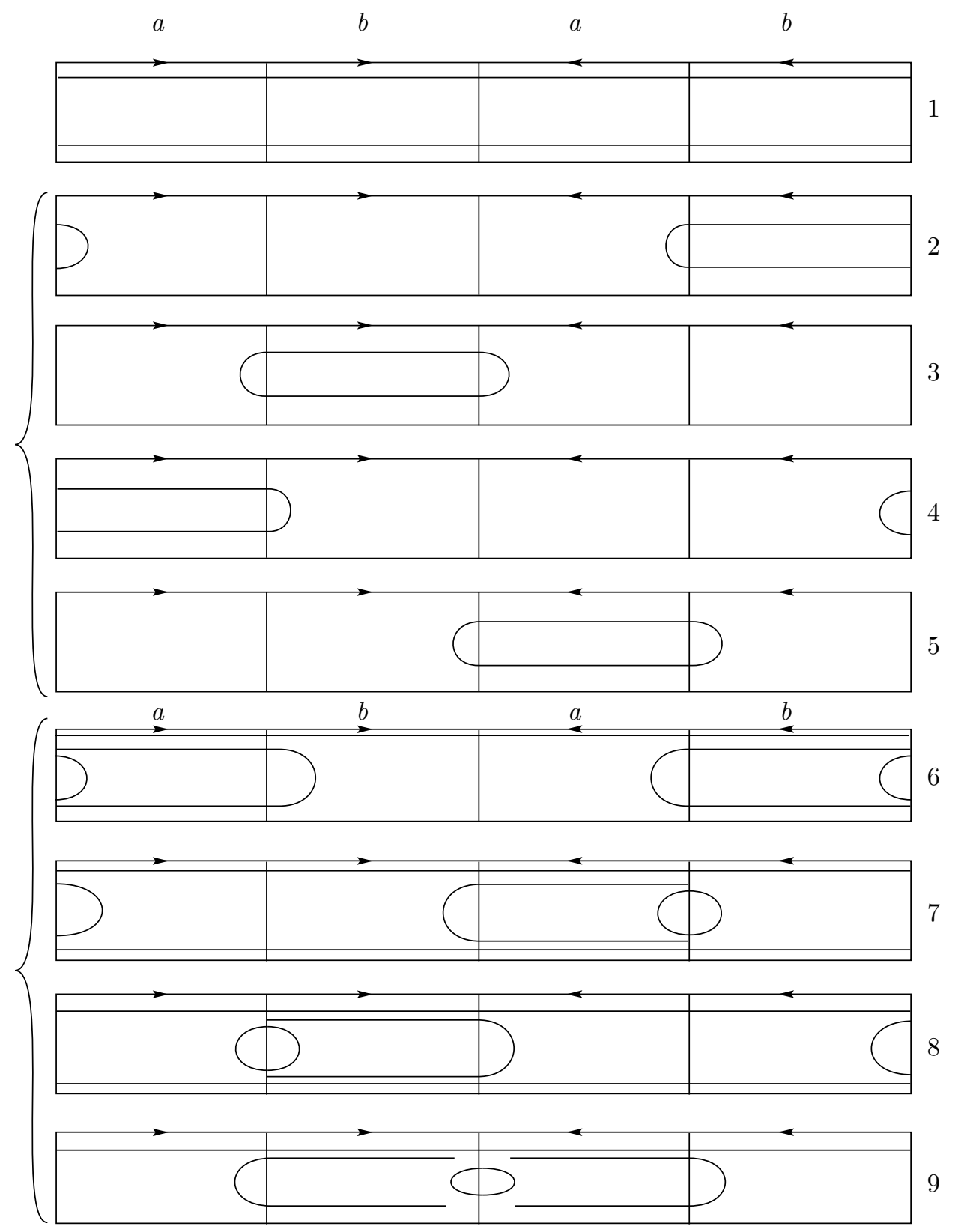

FiguRE 9

$\partial_{v} N(B)$, we can conclude that $M \mid \mathcal{L}$ consists of $I$-bundles, which can be foliated transverse to the $I$-fibers, extending $\mathcal{L}$ to a foliation of $M$ without Reeb components.

For many of the cases, this process is immediate, and the laminations that we have built are easily recognized, see Figure 11a. These laminations, coming from the second through fifth patterns, are clearly the product of a Reeb foliation of an annulus with $S^{1}$. In the remaining four cases (Figure 11b), by assembling the 


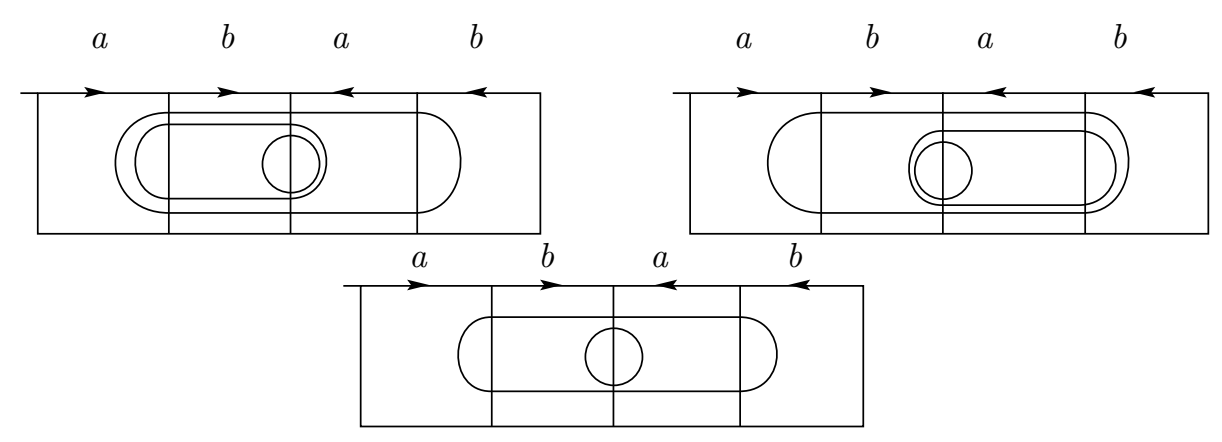

Figure 10
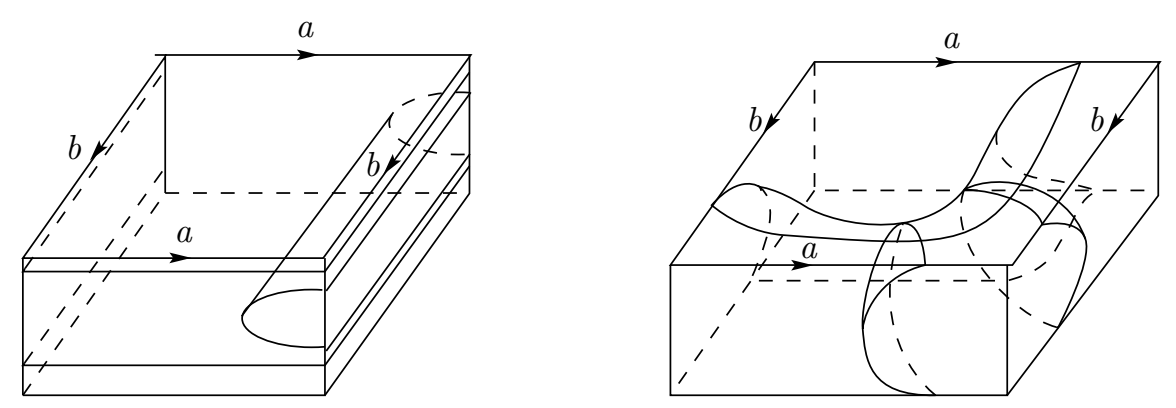

Figure 11

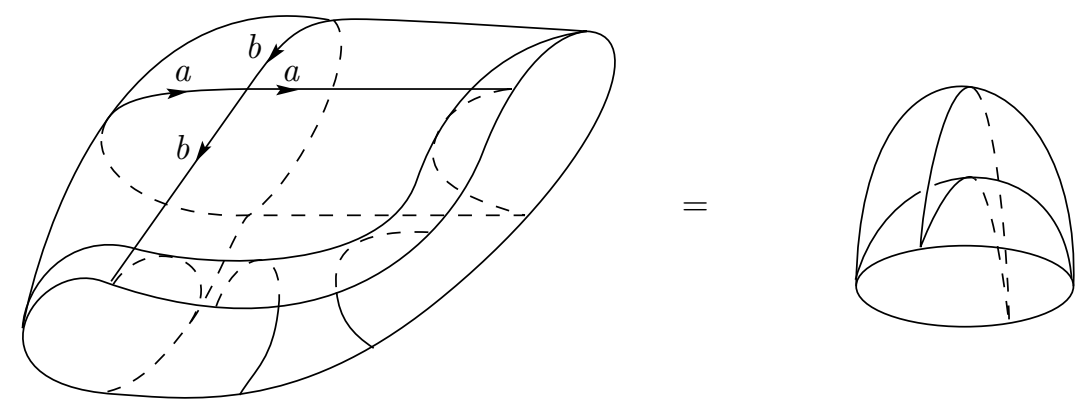

FIGURE 12

pieces of the complement of $B$ in $B^{3}$ together, we can see that the complement of $B$ in $T^{2} \times I$ is a product 3-ball; see Figure 12. Alternatively, one can simply see that this complement is the union of 3-balls glued together, inductively, along 2-disks, and hence is a 3-ball; and by tracing out the arcs which together form the branch curves, we can easily see that there is only one branch curve, and therefore our 3-ball is a product. These laminations are also products of Reeb annuli with $S^{1}$ -our cell decomposition, in some sense, simply chose the wrong 'coordinates'.

Therefore, in every case we can extend our essential lamination to a foliation, proving our first theorem, for $M=T^{2} \times I$. 


\section{The Basic CONTRAdiction}

Before continuing on to consider the higher genus cases, we will now describe how, using the pictures developed in the last section, we will derive our contradictions, by finding 'compressing disks' for our essential lamination $\mathcal{L}$.

We start with an essential lamination $\mathcal{L}$, isotoped to the form described above, so that it meets the 3 -cell in a finite number of families of (normally) parallel disks, each meeting a vertical 1-cell at most twice. $\mathcal{L}$ is carried with full support by a branched surface $B$, which is obtained from $\mathcal{L}$ in the usual way, by collapsing each family of (normally) parallel disks to a single disk, as in Figure 13. Furthermore, in terms of this branched surface, $\mathcal{L}$ still has monogon number $\epsilon$ w.r.t. the 1 -skeleton of $M$; this is because all points of $\mathcal{L} \cap K^{(1)}$ are within $\epsilon$ of the boundary of $M$ (we are assuming that the length of our $I$-factor is greater than $3 \epsilon$ ). All of the branch curves of $B$ intersect $\partial B^{3}$, and so every component of $\partial_{v} N(B)$ contains an $I$-fiber which is part of a vertical arc in one of the vertical 2-cells.

Proposition. If $\mathcal{L}$ and $B$ are as above, then it is not possible to have a loop $\gamma$ in $\partial_{h} N(B)$ bounding a disk $D$ in $M_{0}=M \backslash \operatorname{int}(N(B)$ ) which intersects transversely, exactly once, an arc $\alpha$ in $\partial_{h} N(B)$, joining two (possibly distinct) components of $\partial_{v} N(B)$.

The proof of our second theorem will proceed, in large part, by showing that in the presence of most of the configurations described in the previous section, we can find such a disk and arc.

Proof. Suppose such a disk and arc existed. Then since $\mathcal{L}$ is essential and $\partial_{h} N(B) \subseteq$ $\mathcal{L}$, there exists a disk $D^{\prime}$ in a leaf $L$ of $\mathcal{L}$, with boundary $\gamma . \quad D \cup D^{\prime}=S$ is an embedded sphere in $M$, intersecting $\mathcal{L}$ only in $D^{\prime}$. Because $M$ is irreducible, $S$ bounds a 3-ball $\Delta$ in $M$, and since $S \cap \mathcal{L}=D^{\prime}, \Delta \cap \mathcal{L}=D^{\prime}$ as well, since otherwise $L \subseteq \Delta$ - no leaf of an essential lamination can lie entirely in a 3-ball.

Label the vertical annuli of $\partial_{v} N(B)$ that $\alpha$ joins $A_{1}$ and $A_{2}$ (these may be different labels for the same annulus). $D$ splits $M_{0}$ into components, and, since $\Delta \cap \mathcal{L}=D^{\prime}, \Delta$ must contain one of the components which meets $D$ (see Figure 13). In particular, $\Delta$ must meet, hence contain, one of the vertical annuli, say $A_{1}$.

But since $\Delta$ meets $\mathcal{L}$ only in $D^{\prime}$, it then also follows that both ends of the annulus $A_{1}$ are contained in $D^{\prime}$. But this is absurd; any arc $\beta$ in $D^{\prime}$ joining the ends of

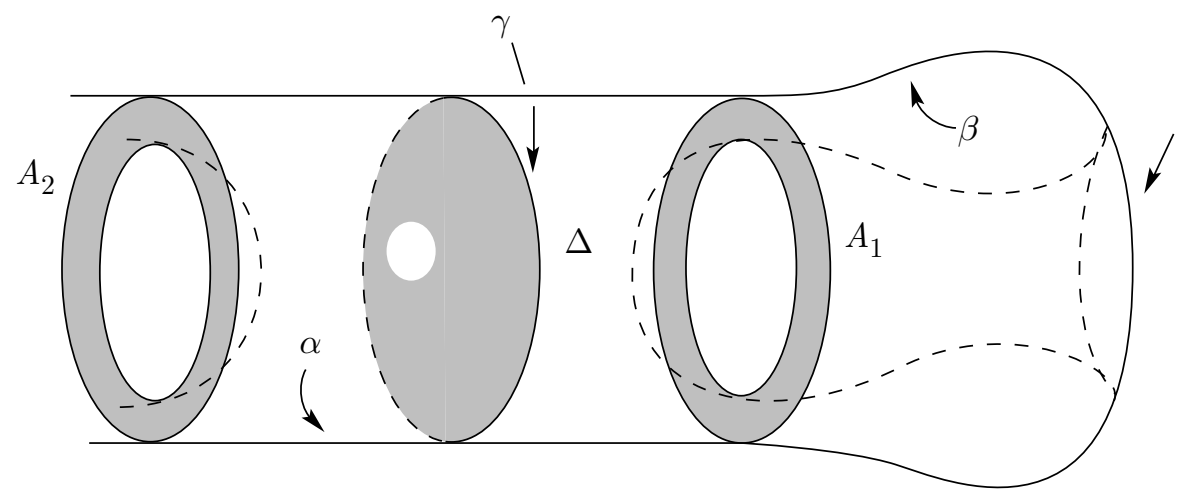

FiguRE 13 
one of the $I$-fibers of $A_{1} \cap K^{(2)}$, together with the $I$-fiber $J$, forms a loop in $\Delta$, which is therefore null-homotopic in $M$, and are therefore homotopic rel endpoints. By extending $\beta$ by adding onto our null-homotopy a vertical rectangle in $N(B) \mid \mathcal{L}$ from $J$ to an arc of $N(B) \cap K^{(1)}$, and letting this longer arc $\beta$ flow back along the isotopies of $\mathcal{L}$ (which leaves its endpoints fixed, because they are stable) we get an arc in our original lamination which is homotopic rel endpoints to an arc of an $I$-fiber in our original $N(B)$, contradicting [G-O, Theorem 1(d)].

\section{THE HIGH-GENUS CASE}

If our surface $F$ has genus $>1$, then after a sufficient number of isotopies of our essential lamination $\mathcal{L}$, we have, around the boundary of our 3-cell, $g$ copies of the various patterns of intersection described in the previous section, strung together in

a circle. We will call each sequence of 4 vertical 2 -cells, corresponding to a sequence $a_{i}, b_{i}, a_{i}^{-1}, b_{i}^{-1}$, a place. What we will now show is that, because $g$ is greater than 1 , unless we have the first pattern at every place, and so have a horizontal lamination, we can always derive a contradiction.

So suppose, by way of contradiction, that in one of the places we have an obvious $\partial$-compressing disk. We therefore have disks of $\mathcal{L} \cap B^{3}$ other than horizontal disks. These disks we will call vertical disks, since they are isotopic to disks in the vertical part of $\partial B^{3}$. The first thing that we must notice is that somewhere in $\partial B^{3}$ there is a vertical 1-cell which is not crossed by any of these vertical disks. This is a consequence of the previous lemma, which showed that no vertical 2-cell contains $\partial$-compressing arcs on both sides. For then if both vertical 1-cells in the boundary of a vertical 2-cell are crossed by vertical disks, then one of the vertical disks must in fact cross both ends; see Figure 7. So if all 1-cells are crossed by vertical disks, then if we shade in all of the disks in the vertical boundary of $B^{3}$ that these vertical disks cut off, the union of these disks form a shaded region $R$ which includes an annulus running completely around $\partial B^{3}$. This shaded region is also a closed set, since the set of loops of $\mathcal{L} \cap K^{(2)}$ null-homotopic in the annulus of vertical 2-cells is closed. But this situation is absurd; the boundary of this region consists of a finite number of horizontal arcs, which must therefore (since $R$ is closed) together form horizontal loops of $\mathcal{L} \cap \partial B^{3}$, which are certainly not null-homotopic in the vertical annulus.

What we will show now is that, in the vertical boundary of the 3 -cell $B^{3}$, certain of the patterns (2) through (9) cannot occur, either singly or in combination with others. In the end, we will so restrict the boundary behavior of $\mathcal{L} \cap B^{3}$ that only one possibility will remain - that all of the places have patterns of type (1). In each case, we will, after assuming that a certain sequence of pattern types occurs, derive our contradiction in one of two ways: either we will find two loops in $\partial_{h} N(B)$ which intersect in a point, one of which is null-homotopic in $M$, or we will find a loop and arc as described in the proposition above. Each gives a contradiction; in the first case, this is because the null-homotopic loop must be null-homotopic in the leaf which contains it, but the intersection condition implies that it is in fact homologically non-trivial in that leaf. The second case is our proposition. The basic idea is that if the 'complicated' pieces are relatively sparse, it is fairly easy to find the first kind of behavior, while if the complicated pieces are more prevalent, we tend to find the second kind of behavior. 
Lemma. If $\mathcal{L} \cap \partial B^{3}$ contains vertical disks, then every vertical 1 -cell $J$ of $B^{3}$, in the interior of one of the places of four vertical 2-cells, is either not intersected by any vertical disk of $\mathcal{L}$, or is intersected by an obvious $\partial$-compressing arc in one of the two adjacent vertical 2-cells of $\partial B^{3}$ that contains it.

Proof. Suppose there were a 1-cell both intersected by a vertical disk, and missed by our obvious $\partial$-compressing arcs. Our situation would be like Figure 14a. Then in $B^{3}$ there is a $\partial$-compressing disk for the outermost of these vertical disks, intersecting $\partial B^{3}$ in a subarc of $J_{0}$ of $J$ with $\mathcal{L} \cap J_{0}=\partial J_{0}$. This disk, together with the obvious $\partial$-compressing disk which we know exists, forms a disk $D$ whose boundary lies in $\partial_{h} N(B)$. If one of the 2-cells adjacent to $J$ does not contain any obvious $\partial$ compressing arc, then the horizontal arc that it does intersect closes up in $F \times I$ to a loop, which lies in $\partial_{h} N(B)$, so $D$ intersects another loop in $\partial_{h} N(B)$ exactly once, a contradiction; see Figure 14b. If both 2-cells have an obvious $\partial$-compressing arc, then $D$ intersects exactly once an arc running between two components of $\partial_{v} N(B)$, contradicting the proposition; see Figure 14c.

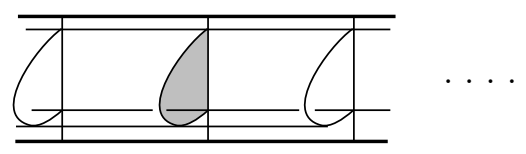

(a)

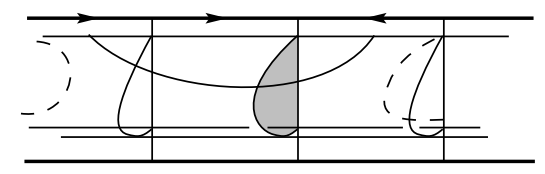

(b)
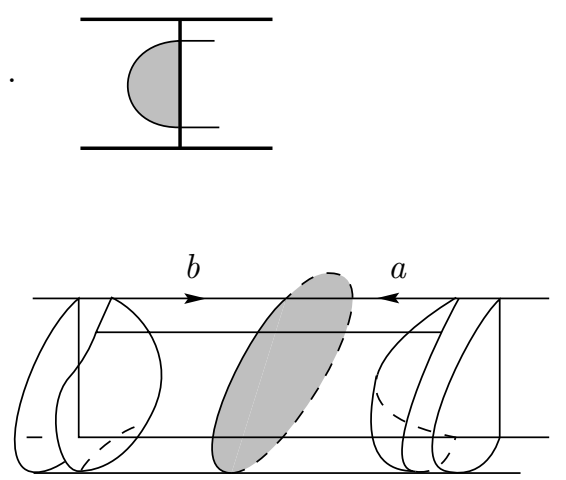

(c)

FiguRE 14

With the lemma we can begin to eliminate some of the pattern types which we have listed in the $T^{2} \times I$ case above. The first four of these remaining types, ' 2 ' through ' 5 ', are fairly easy to deal with. In each of these we have a vertical 2 -cell, which contains no obvious $\partial$-compressing arcs, yet is crossed by a vertical disk of $\mathcal{L} \cap B^{3}$. If, everywhere else around the boundary of the 3 -cell $B^{3}$, we have no obvious $\partial$-compressing arcs, then our situation is like the one pictured in Figure 15 ; there is only one family of vertical disks, and they fail to intersect one of the vertical 2-cells. We must then be dealing with either type ' 3 ' or ' 5 ': the other two types would give us a situation violating the lemma above, since this family of vertical disks would cross an entire place of 2-cells (hence one of its interior 1-cells). Then it is an easy matter to construct a compressing disk $D$ for $\mathcal{L}$; this is a 'real' compression, because we can find another loop in the same leaf which intersects $D$ exactly once, using the fact that the vertical disk misses one of the 2-cells.

On the other hand, if one of the other places does have a $\partial$-compressing arc, then, for the types ' 3 ' and ' 5 ', we can glue the three 'corners' of our $4 g$-gon (that the three central vertical arcs of our picture represent) together, and draw how 


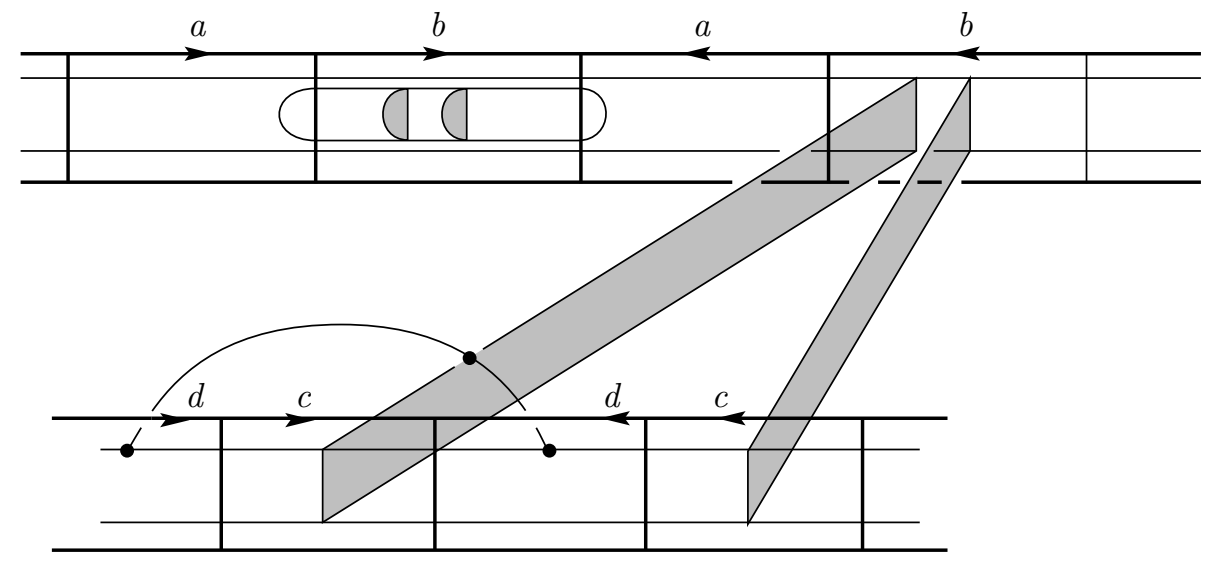

FIGURE 15

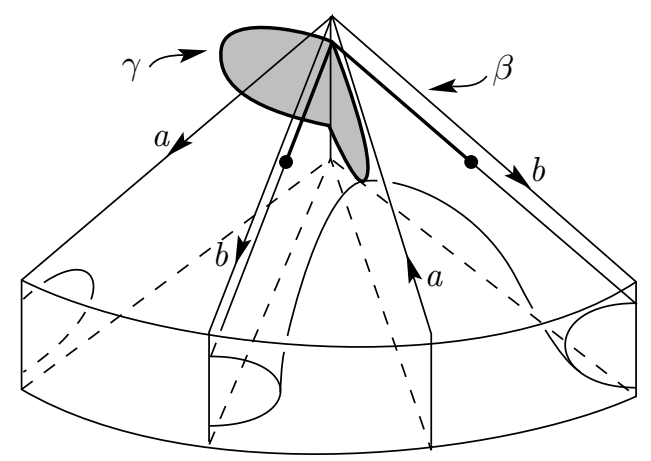

FIGURE 16

our lamination intersects this 3-fold corner; see Figure 16. We can then glue the obvious $\partial$-compressing disk, lying in the interior of this 3 -fold corner, to the disk that the other $\partial$-compressing arc cuts off, to obtain a compressing disk for $\mathcal{L}$ (see Figure 16); the two vertical arcs that these $\partial$-compressing disks cobound are really the same, living in the single vertical 1-cell of $M$. This compressing disk is 'real', since the horizontal arc $\beta$ in the 3 -fold corner lying in the vertical disks and furthest from the top (but still within $\epsilon$ of the top) forms a closed loop in $M$, which lies in $\partial_{h} N(B)$ and intersects our compressing disk, transversely, exactly once.

For types ' 2 ' and ' 4 ', there are two possibilities. One of the 2-cells (in our type ' 2 ' or ' 4 ' place) having no obvious $\partial$-compressing arcs is crossed by a vertical disk; if both 2-cells are, then we can build a compressing disk and a loop in $\partial_{h} N(B)$ intersecting it, transversely, once; see Figure 17a. If the second 2-cell $R_{1}$ in our type ' 2 ' or ' 4 ' place is not crossed by a vertical disk, then there are still two possibilities. If the pattern of the place which includes the second $\partial$-compressing arc is not type ' 9 ' (note that types ' 3 ' and ' 5 ' have already been eliminated), then the place has an interior 1-cell which doesn't meet any obvious $\partial$-compressing arc, and therefore, by the lemma, is not crossed by a vertical disk. Therefore there is a vertical 2-cell $R_{2}$ in that place which isn't crossed by a vertical disk, but the 2-cell it is glued to is. 

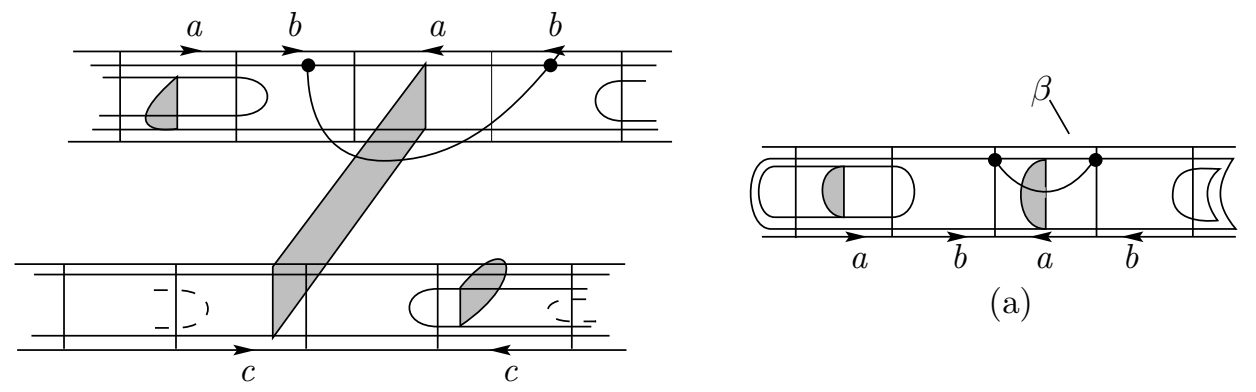

(a)

(b)

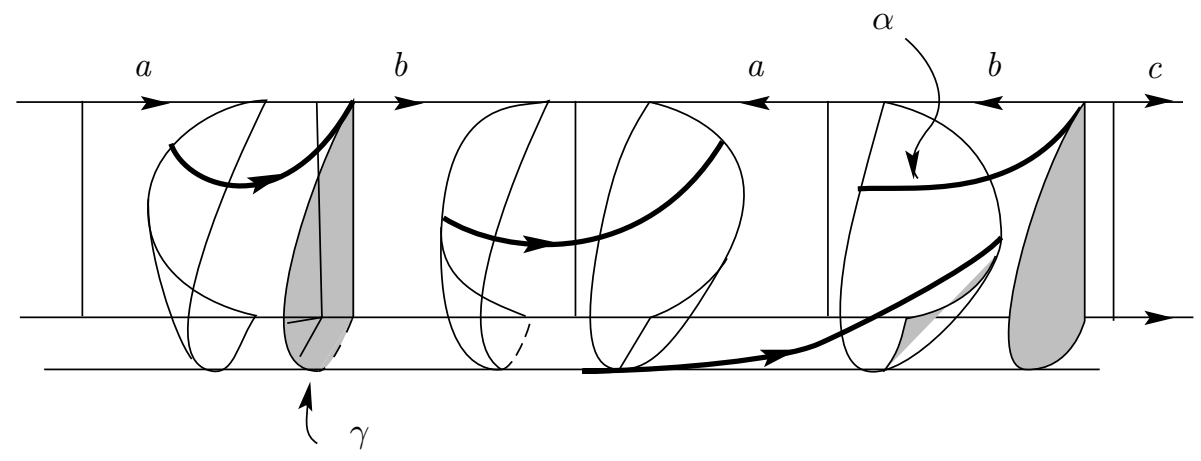

(c)

FiguRE 17

By drawing an arc in the horizontal disks from $R_{1}$ to $R_{2}$, and taking the vertical rectangle lying below it, and then adding two $\partial$-compressing caps, we can create a compressing disk (Figure 17b) which is intersected by a loop in $\partial_{h} N(B)$ exactly once.

Finally, if all of the remaining places have type ' 1 ' or ' 9 ', then since the vertical disks originating from our original (type (2) or (4)) place continue out from the end of the place, the first type ' 9 ' place we meet starting from either end must have a vertical disk crossing at least one of its ends. This gives us a $\partial$-compressing disk, at one end, which, together with another (obvious) one from the interior of the type ' 9 ' place, gives us a compressing disk for which we can find an arc joining branch curves, intersecting our compressing disk exactly once; see Figure 17c. This, however, violates the proposition.

Consequently, none of the places around the boundary of the 3-cell have any of the patterns ' 2 ' through ' 5 '.

This leaves four remaining types, ' 6 ' through ' 9 '. First, if we glue the three 'corners' of each pattern (6) through (9), that the three central vertical arcs of each pattern represent, together, and draw the portions of our branched surface $B$ that live near this 3 -fold corner, then we get the four figures of Figure 18. Note that 

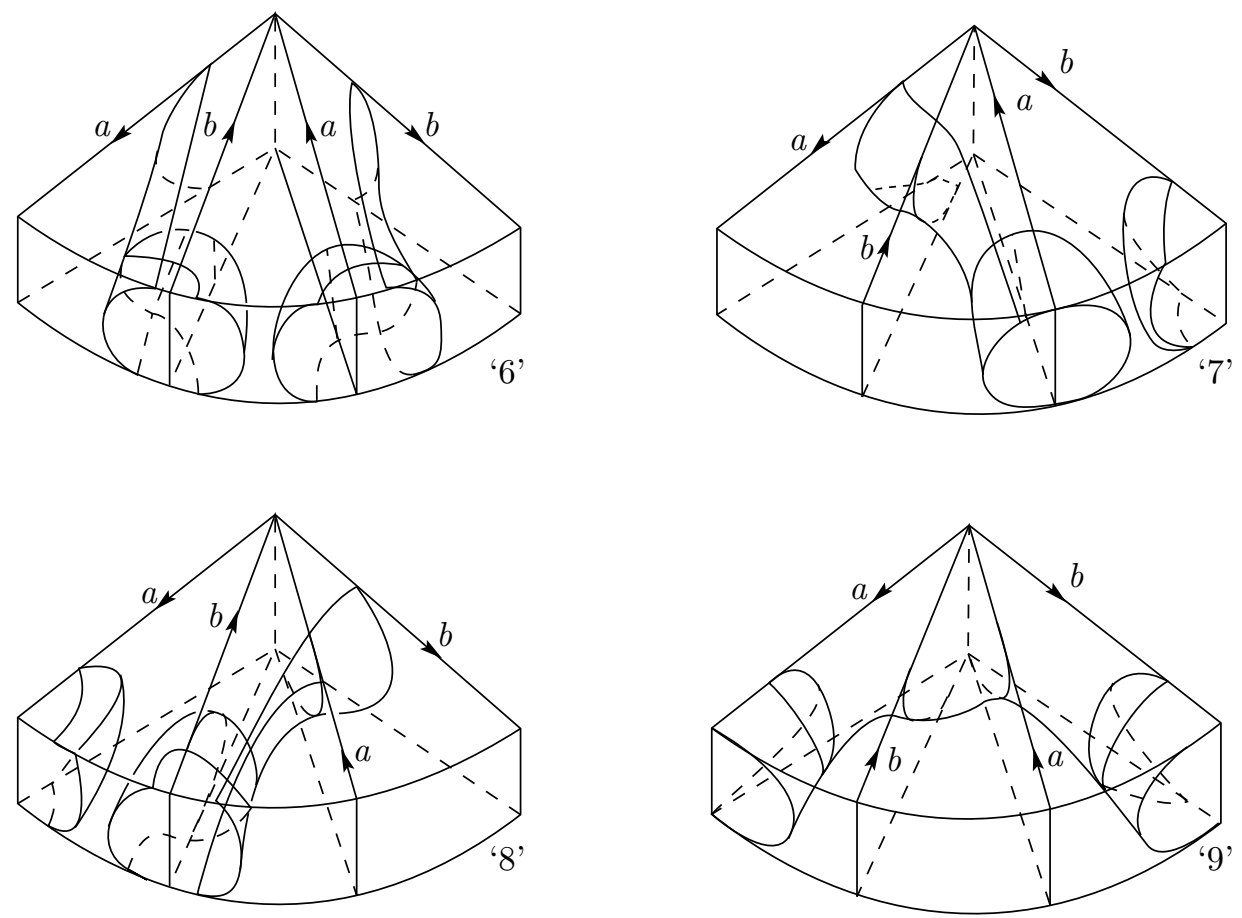

Figure 18

each of these corners have obvious $\partial$-compressing arcs on both ends. Between each adjacent pair of 3 -fold corners, there is also a 'transitional corner'. The shape of the branched surface $B$ in these transitional pieces is determined by how the branched surface intersects each end; in addition to the case that $B$ consists only of the top and bottom horizontal faces of the corner, we have one of 8 possibilities, see Figure 19.

We will deal first with the possibility of a type ' 6 ' place, from Figure 9 . There is a fairly obvious compressing disk $D$ in its 3 -fold corner, formed (across the vertical 1cell) from the two $\partial$-compressing arcs on either side of the 3 -fold corner (see Figure 18). Because in our present situation the transitional pieces (b) and (d) cannot occur adjacent to our chosen corner (they imply that the next place over is a type (1) place with a vertical disk crossing it, contradicting the lemma), it is easy to see that if there is another $\partial$-compressing arc in one of the other places, then we can join our compressing disk to a branch curve by an arc, outside of our 3 -fold corner, in $\partial_{h} N(B)$. In every case but (a), this can be done in the adjacent transitional piece; in the remaining case, it can be done immediately upon entering the next 3 -fold corner (which must be the left or right end of ' 6 ', the left of ' 7 ', or the right of ' 8 '). This together with the obvious arc in our original type ' 6 ' corner joining $D$ to a branch curve in the 3 -fold corner, gives an arc satisfying the conditions of our proposition, a contradiction.

Similarly, the last of the types, type ' 9 ', cannot occur. If there were any other obvious $\partial$-compressing arc around the boundary of $B^{3}$, then by gluing it to the $\partial$-compressing arc in the middle of our 3 -fold corner, we would get a compressing 

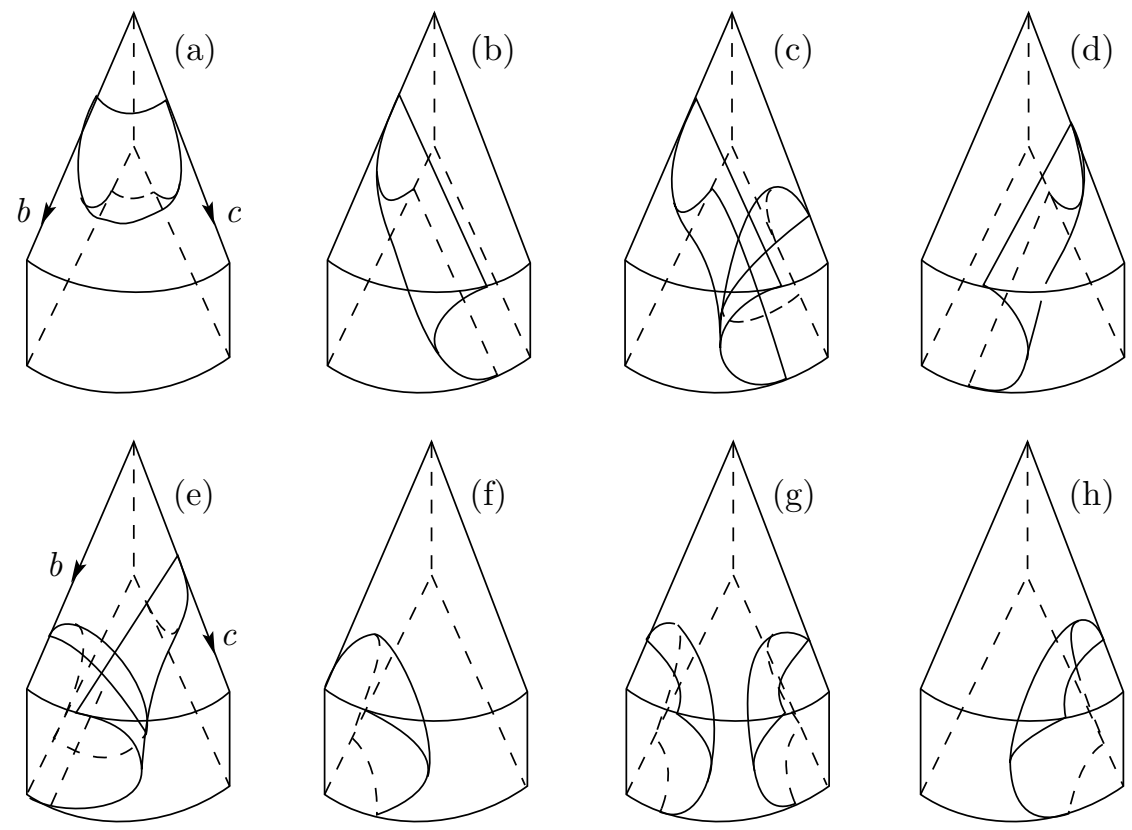

FigURE 19

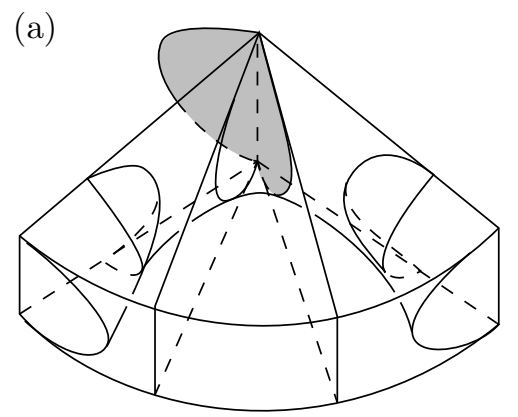

(b)

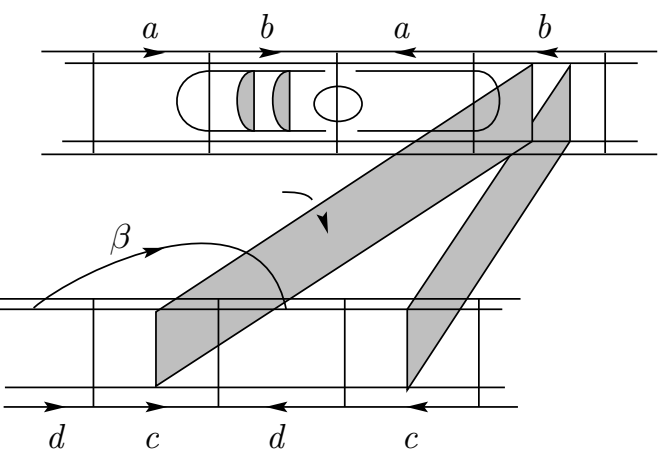

FiguRe 20

disk $D$; see Figure 20a. The obvious arc in the neighborhood of the 3 -fold corner joining the two branch curves intersects $\partial D$ transversely exactly once, contradicting the proposition. On the other hand, if there are no other $\partial$-compressing arcs in the boundary of $B^{3}$, then we have the situation in Figure 20b; the only vertical disks then live inside of our single type (9) place. We can then build a different compressing disk for $B$, by chosing the vertical rectangles lying over the arcs shown in Figure 20b, whose boundary intersects a loop $\beta$ in $\partial_{h} N(B)$ transversely in a single point. This again is a contradiction, so the last of the four remaining types also cannot occur.

This leaves only the two middle types, types ' 7 ' and ' 8 ', remaining. Both of these patterns have a 1-cell not meeting an obvious $\partial$-compressing arc, so the adjacent 2 -cells are not completely crossed by vertical disks, by the lemma. Also, at least one 


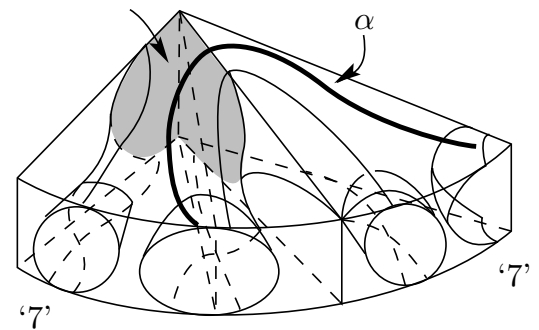

(a)

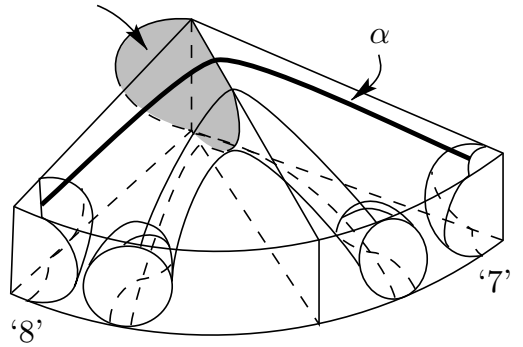

(b)

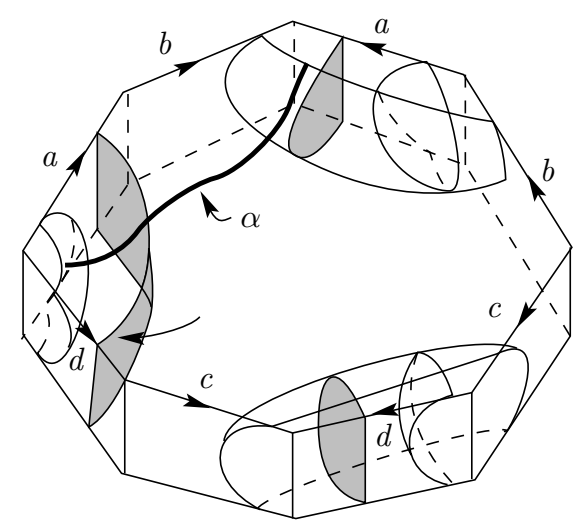

(c)

FIGURE 21

of the remaining two (end) 2-cells in each pattern is (obviously) crossed by a vertical disk. As with the previous two types, if there is a place around $\partial B^{3}$ which has a type (1) pattern (i.e., has no obvious $\partial$-compressing arcs), then that place would either be crossed by an outermost vertical disk, contradicting the lemma, or is not crossed by any vertical disk, allowing us to build a compressing disk intersecting another loop exactly once; this is entirely similar to Figure 20b. Therefore, we may assume that in none of the places around $\partial B^{3}$ have a type (1) pattern; all of them are type $(7)$ or $(8)$.

But now let us look in a neighborhood of our single vertical 1-cell in $M$; this is made up of our (type (7) or (8)) 3-fold corners, together with transitional pieces in between each. What we will find is that no matter what sequence of type (7) or (8) corners we fit together around our 1-cell, we can always find a compressing disk and arc satisfying the hypotheses of the proposition. Finding a disk is easy; each 3 -fold corner contains an obvious $\partial$-compressing disk to act as 'half' of a compressing disk. The problem is that in both of these remaining types, these halves are at the edges; they don't obviously separate two branch curves, as did the type (6) and (9) cases. However, it is not hard to see that if two places of the same type occur side-by-side, then the resulting compressing disk does separate a pair of branch curves, and so we can find the extra arc we require to appeal to the proposition; see Figure 21a. Two type ' 7 ' places are shown there; type ' 8 ' is the mirror image. 
This leaves only the possibility that these two types alternate as we travel around the boundary of the 3-cell $B^{3}$, and therefore there are an even number of places in the boundary. If there are more than two places, we can once again find a compressing disk separating branch curves, by gluing half-disks from two non-adjacent places together; see Figure 21b. Finally, if there are only two places, then we can draw exactly what $B$ looks like in $B^{3}$, since we know that the 1-cells in this figure that are not met by a $\partial$-compressing disk are also not crossed by a vertical disk; see Figure 21c. Then we can find a compressing disk and arc which satisfies the hypotheses of the proposition.

This covers all of the possibilities, under the assumption that $\mathcal{L} \cap \partial B^{3}$ contains a $\partial$-compressing arc. In all of them, we found a disk $D$ in $M \backslash N(B)$ with $\partial D=\gamma \subseteq \partial_{h} N(B)$, together with either a loop in $\partial_{h} N(B)$ intersecting $\gamma$ transversely in a single point, or an arc in $\partial_{h} N(B)$ joining two components of $\partial_{v} N(B)$ and intersecting $\gamma$ transversely in a single point. But both of these situations contradict the fact that $\mathcal{L}$ is essential. Therefore, there can be no $\partial$-compressing arcs; so $\mathcal{L}$ can be made horizontal in $M=F \times I$.

\section{The most General SituAtion}

We have dealt with the case that $M=F \times I$, where $F$ is a closed orientable surface. We now turn our attention first to the case that $M=F \times I$, where $F$ is a closed non-orientable surface. Then, as we described before, $F$ can be represented as a $2 g$-gon whose edges are labelled counterclockwise $a_{1}, a_{1}, \ldots, a_{g}, a_{g}$. The same collection of isotopies, if it doesn't make $\mathcal{L}$ horizontal, will, we can assume, concentrate the unstable points within $\epsilon$ of $\partial M$. We get a collection of vertical disks, whose boundaries each cross a vertical 1-cell at most twice. The resulting collection of $\partial$-compressing arcs are found on at most one end of each vertical 2-cell, and no horizontal arc can run from the top of a 2-cell to the bottom. Around the boundary of the 3-cell $B^{3}$ we therefore have g pairs of (length 2) places, and in each we have one of three patterns, shown in Figure 22.

In the case of $K^{2}$, we have two of these places. If we do not have a horizontal lamination, then we must have $\partial$-compressing arcs in each pair, pointed in the opposite directions; any other possibility would not give us any vertical disks (i.e., there would be holonomy around the union of the upper, say, boundaries of the vertical 2-cells). But it is then easy to see that the obvious branched surface that we build out of these disks has complement which is a product, and so the lamination can be completed to a foliation; see Figure 23.

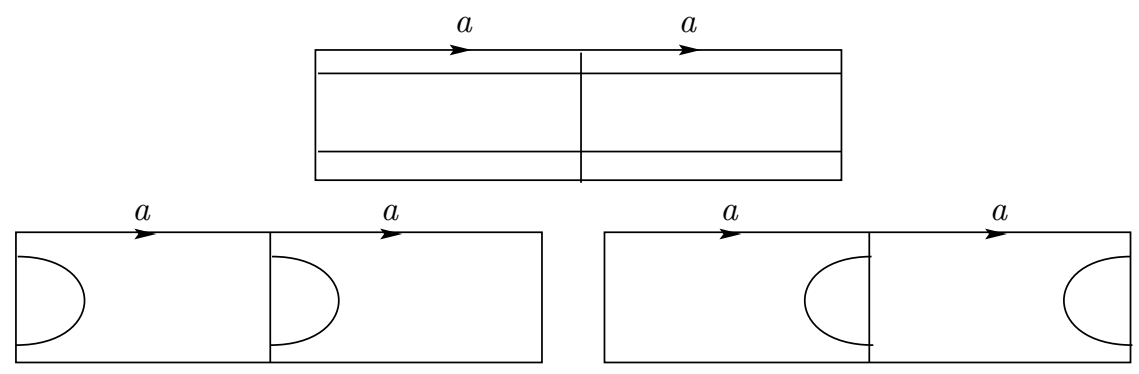

Figure 22 


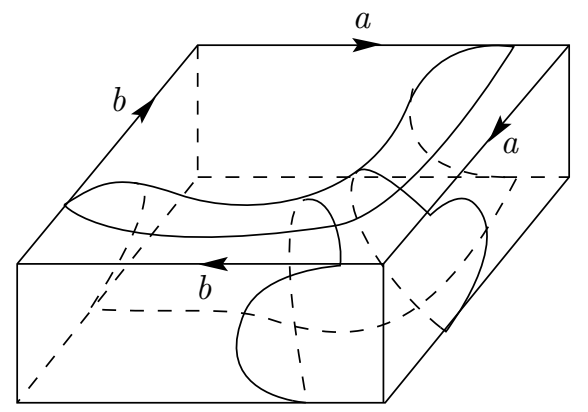

(a)

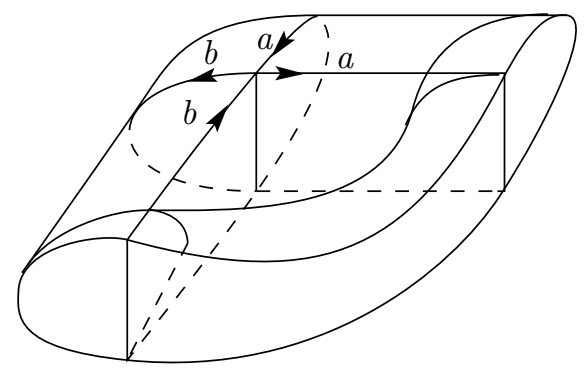

(b)

FigURE 23

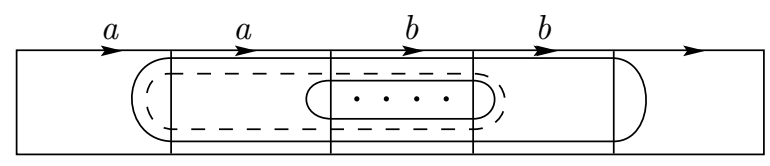

(a)

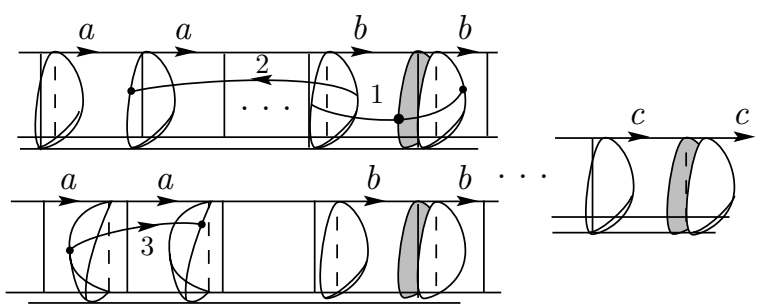

(b)

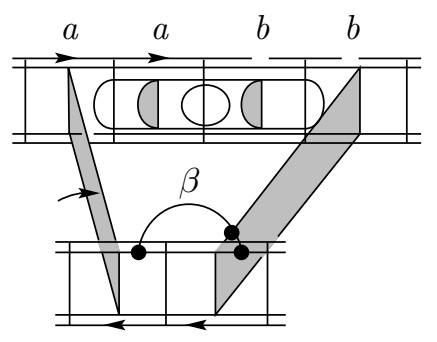

(c)

FiguRE 24

In the higher genus cases, we have three or more places around the boundary of $B^{3}$, and, again, if we have any $\partial$-compressing arcs, we must have at least two sets, pointed in opposite directions. We can assume, as we travel in the counterclockwise direction, that they open towards one another; see Figure 24a. If there is a collection of horizontal arcs in between, then they are crossed by a vertical disk, contradicting the lemma. If there is a third collection of $\partial$-compressing arcs, it opens up in the same direction as one of the previous two; then their two obvious $\partial$-compressing arcs together form a compressing disk, for which we can find an arc required for the proposition; see Figure 24b. There are two cases, depending upon whether the next place adjacent to one of the two also points in the same direction or not.

Therefore, we must have exactly two sets of $\partial$-compressing arcs, in adjacent strips and opening toward one another. The remaining strips then have only horizontal arcs running across them, and therefore have no vertical disks crossing them, by 
the lemma. Then we can find a compressing disk $D$ and a loop intersecting $\partial D$ transversely in one point, as in our previous type (9) case; see Figure 24c.

So in all cases, except when there are only horizontal disks (and therefore a horizontal lamination), we arrive at a contradiction. So every essential lamination in $F \times I$, where $F$ is a closed surface of genus greater than one, can be isotoped to a horizontal lamination.

Finally, we deal with the case that $M$ is an $I$-bundle over a closed surface $F$, but not necessarily a trivial $I$-bundle. This means that $M$ is obtained by gluing a $4 g$-gon crossed with $I$, or a $2 g$-gon crossed with $I$, together using the identifications for the base surface, except some of the gluings flip the $I$-direction of the vertical 2cells. This flipping does alter the proofs that we have given for the trivial $I$-bundle cases, although largely in a minor way. This is because all of the compressing disks we have found in the course of our constructions have been vertical compressing disks, as the reader can speedily check. This means that they can still be built, even if some of the gluings flip the $I$-direction.

The isotopy construction we have given goes through without any change, so we may, as in the previous cases, assume that the usual properties hold. So after isotoping $\mathcal{L}$, all of points of $\mathcal{L}$ in the vertical 1-cell are within $\epsilon$ of $\partial M, \mathcal{L} \cap B^{3}$ consists of a finite number of parallel families of disks, each of which crosses each vertical 1-cell at most twice, each vertical 2-cell has at most one parallel family of $\partial$-compressing arcs, and no horizontal arc in a 2-cell runs from the top on one side to the bottom on the other.

For the cases when the base of the bundle is $T^{2}$ or $K^{2}$, we can still easily see that the lamination can be extended to a foliation. The pieces of the complement of $B$ in $B^{3}$ still glue together to give a collection of $I$-bundles (and most of these are still product $I$-bundles), so we can fill in these complementary pieces with (horizontal) foliations.

In the higher genus cases, most of the constructions of compressing disks for the non-type (1) cases will go through unchanged. In particular, any compressing disk $D$ that came equipped with an arc joining branch curves, and which crossed $D$ once, will still exist, as the reader can readily verify. This leaves the cases which we dealt with by finding a compressing disk $D$ and a loop intersecting $\partial D$ transversely in a single point. Some of these must be dealt with slightly more carefully, since we were tacitly using the fact that the gluings did not flip the $I$-directions.

However, if we assume that the boundary $\gamma$ of the compressing disk $D$ that we constructed in those situations bounds a disk $D^{\prime}$ in the leaf $L$ of $\mathcal{L}$ that contains it (which we can; $\mathcal{L}$ is essential!), then we can create a slightly different contradiction. Any loop $\beta$ that we find in $L$ which is transverse to $\gamma$ then intersects $D^{\prime}$ in $\operatorname{arcs}$, and so if we orient $\beta$ and an annular neighborhood of $\gamma$ (it is annular because $\gamma$ bounds a disk in $L$ ), then the intersection number of $\beta$ with $\gamma$ is zero, where this number is counted by comparing the orientations of the loops with an orientation of the annulus.

But now in each of the cases above where the contradiction was obtained by finding an additional loop (rather than arc) we can find a loop with non-zero intersection number. In the case of Figure $14 \mathrm{~b}$ of the lemma, we choose instead a loop as in Figure 25a; this loop has intersection number 2 with the boundary of the compressing disk. In the cases analogous to Figures $15,17 \mathrm{~b}, 20 \mathrm{~b}$, and $24 \mathrm{c}$, we find the loop instead, if necessary (i.e., if the 2-cell we used was glued with a flip), by 
taking both a 'top' and 'bottom' arc, which together form a single loop which has intersection number 2 with the boundary of the disk; see Figures $25 \mathrm{bc}$.

So in the higher genus cases, we can still find all of the required compressing disks needed to obtain our contradictions; every essential lamination can be isotoped to a horizontal one.

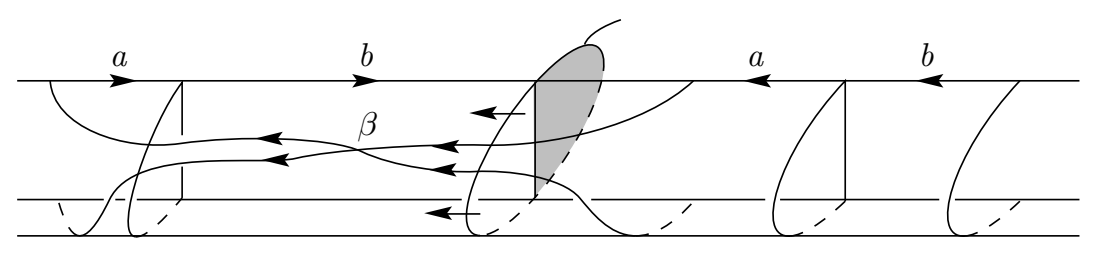

(a)

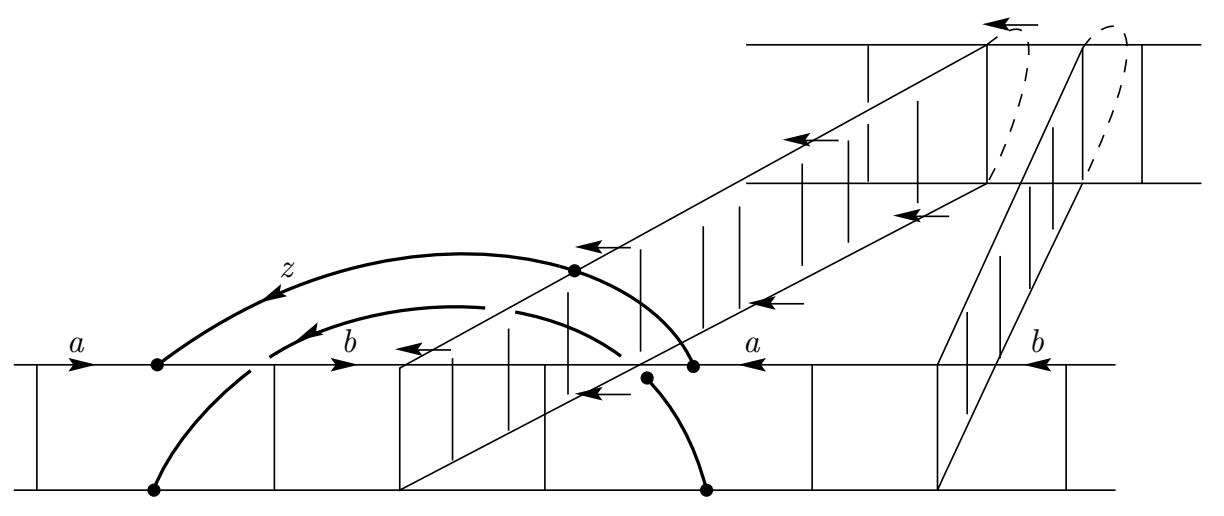

(b)

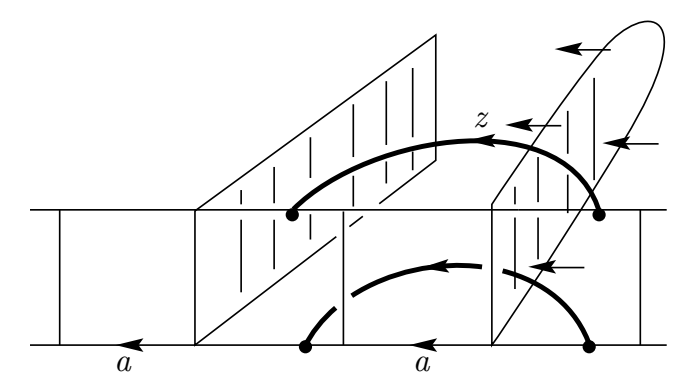

(c)

FiguRe 25 


\section{Concluding Remarks}

The techniques used in this paper also give a simplified proof of the main result of [B3]-Haken normal form for regular cell decompositions. Instead of using a sequence of infinite isotopies, we build a single infinite isotopy, as we did here, by making the lamination $\mathcal{L}$ meet each 3 -cell in disks and then, in turn, pushing all of the identifiable $\partial$-compressing disks out of each 3 -cell, not worrying if some of them push problems back into the 3-cell. This sequence of isotopies only erases points of intersection with the 1-skeleton (which is in some sense the crucial fact in all of these proofs), giving us a non-empty set of stable points. Then the argument outlined here implies that stable disks grow out of these stable points; we can then finish by using the arguments of [B2] to show that the resulting 'splitting of the closure of the union of the stable disks' contains an essential sublamination.

To make these techniques more useful, it would be good to know how to handle the possibility that the intersections of the isotoped laminations with the 1-skeleton is not necessarily nested. In this respect, the paper [B3] offers one such avenue. The possibility that new points can be added to the intersection with the 1-skeleton present the main obstacle to applying techniques like those of this paper to several problems of central importance to the theory of essential laminations. Can one always obtain an essential lamination (or the empty set) by repeatedly surgering certain kinds of laminations; for example, the pullback of an essential lamination under a map between 3-manifolds? A positive answer to this question would settle the conjecture that 3-manifolds containing essential laminations are determined up to homeomorphism by their homotopy type [B4]. Given an essential lamination $\mathcal{L}$ in $M$ and a knot $\kappa$ in $M$, can one find a new essential lamination $\mathcal{L}^{\prime}$ so that $\mathcal{L}^{\prime} \backslash \operatorname{int}(N(\kappa))$ is essential in $M \backslash \operatorname{int}(N(\kappa))$ ? The only obstruction is that there might be compressing disks for $\mathcal{L} \backslash \operatorname{int}(N(\kappa))$ in $M \backslash \operatorname{int}(N(\kappa))$, so it is again an (isotopy through) surgery problem. A positive answer to this question would allow us to conclude that if we can find an essential lamination after Dehn filling along a knot, then there exists one before, which 'survives' the filling. It seems likely that techniques along the lines of this paper sufficient to settle the first of these questions would succeed in settling the second of these, as well.

\section{REFERENCES}

[B1] M. Brittenham, Essential laminations in Seifert-fibered spaces, Topology 32 (1993), 61-85. MR 94c:57027

[B2] M. Brittenham, Essential laminations and Haken normal form, Pacific Journal of Mathematics 168 (1995), 217-234. MR 96c:57028

[B3] M. Brittenham, Essential laminations and Haken normal form: regular cell decompositions, preprint (1991).

[B4] M. Brittenham, Essential laminations and deformations of homotopy equivalences: from essential pullback to homeomorphism, Topology and its Applications 60 (1994), 249-265. MR 95i:57019

[G-O] D. Gabai and U. Oertel, Essential laminations in 3-manifolds, Annals of Mathematics 130 (1989), 41-73. MR 90h:57012

[H] W. Haken, Theorie der Normalflaschen, Acta. Math. 105 (1961), 245-375. MR 25:4519c

Department of Mathematics, Vassar College, Poughreepsie, New York 12601

E-mail address: brittenham@vassar.edu 